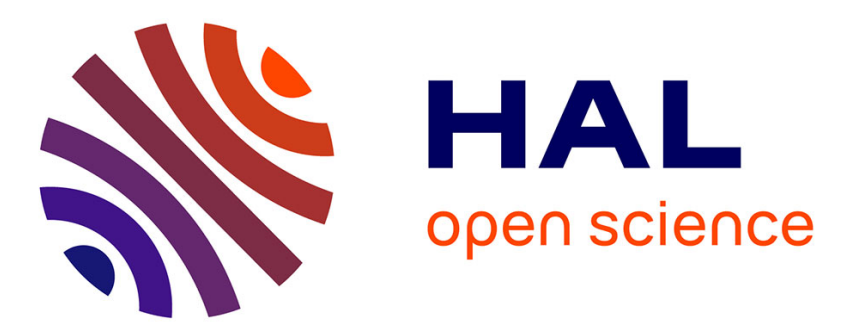

\title{
Using Fuzzy Logic for data priority aware collection in RFID sensing wireless networks
}

Abdoul Aziz Mbacké, Nathalie Mitton, Hervé Rivano

\section{To cite this version:}

Abdoul Aziz Mbacké, Nathalie Mitton, Hervé Rivano. Using Fuzzy Logic for data priority aware collection in RFID sensing wireless networks. PIMRC 2018 - IEEE International Symposium on Personal, Indoor and Mobile Radio Communications, Sep 2018, Bologne, Italy. pp.1-5. hal-01830721

\section{HAL Id: hal-01830721 \\ https://hal.inria.fr/hal-01830721}

Submitted on 5 Jul 2018

HAL is a multi-disciplinary open access archive for the deposit and dissemination of scientific research documents, whether they are published or not. The documents may come from teaching and research institutions in France or abroad, or from public or private research centers.
L'archive ouverte pluridisciplinaire HAL, est destinée au dépôt et à la diffusion de documents scientifiques de niveau recherche, publiés ou non, émanant des établissements d'enseignement et de recherche français ou étrangers, des laboratoires publics ou privés. 


\title{
Using Fuzzy Logic for data priority aware collection in RFID sensing wireless networks
}

\author{
Abdoul Aziz Mbacke \\ Univ Lyon, Inria, INSA Lyon, CITI, France \\ Nathalie Mitton \\ Hervé Rivano \\ aziz.mbacke@inria.fr \\ Inria, France \\ Univ Lyon, INSA Lyon, Inria, CITI, France \\ nathalie.mitton@inria.fr_herve.rivano@insa-lyon.fr
}

\begin{abstract}
Long being used for identification purposes, a new set of applications is now available thanks to the development of RFID technology. One of which is remote sensing of environmental values using passive RFID tags. This leap forward allowed a more energy efficient and cheaper solution for applications like logistics or urban infrastructure monitoring. Nevertheless, serious issues raised with the use of RFID: (i) reading collisions and (ii) gathering of tag information. Indeed, tags information retrieved by readers have to be transmitted towards a base station through a multihop scheme which can interfere with neighboring readers activity. In this paper, we propose cross-layer solutions meant for both scheduling of readers' activity to avoid collisions, and a multihop routing towards base stations, to gather read tag data. This routing is performed with a data priority aware mechanism allowing end-to-end delay reduction of urgent data packets delivery up to $13 \%$ faster compared to standard ones. Using fuzzy logic, we combine several observed metrics to reduce the load of forwarding nodes and improve latency as well as data rate. We validate our proposal running simulations on industrial and urban scenarios.
\end{abstract}

\section{INTRODUCTION}

Radio Frequency Identification (RFID) is a mean of wireless, non-direct line of sight identification. It is based on two main components: tags that hold the information and readers that can access the information stored in tags. Tags can either be passive, relying on the reader's interrogation signal power to enable themselves and respond (backscattering), or active using a battery to initiate communication and transmit their information [1]. With regards to the intended applications, we mainly focus on passive tags working in the frequency band of $865-868 \mathrm{MHz}$ [2]. Such passive tags do not need a battery to function, and this frequency provides longer interrogation ranges up to $12 \mathrm{~m} \mathrm{[2]}$.

Throughout the years, one focus of RFID research has been energy harvesting to provide more than backscattering, like environmental sensing [3]. One solution proposed [4], was to link passive tags to a capacitor that would be charged up by interrogation signals and then be able to power sensors providing temperature and fullness of milk cartons. Once the sensing is performed, the data

This work was partially supported by a grant from CPER/FEDER DATA, NRF, the French ministeries MESRI and MEAE, the LIRIMA Agrinet project and IPL CityLab@Inria. is stored in the tag until the next interrogation round. An enhanced passive UHF sensor tag is also proposed [5], capable of performing sensing upon reading with a valid range of up to $6.5 \mathrm{~m}$. One example is shown [6] where temperature and pressure sensors were attached to RFID passive tags for sensing within industrial applications. Results validated a high level of precision with a fair error margin. Structural Health Monitoring [7] in order to monitor the state of concrete by sensing temperature, humidity and $\mathrm{pH}$-value using embedded passive tags or Solid Waste Monitoring using passive RFID tags [8] are other concrete applications. All these advances motivate the use of passive RFID as a complementary solution to traditional Wireless Sensor Networks (WSN) for smart cities and industrial applications. Nevertheless, a few issues still need to be overcome in order to fully use RFID for IoT [9], such as collisions issues and connecting RFID systems to the back-end infrastructure. The first issue is observed when multiple readers attempt to interrogate the same tags within their range generating collisions and preventing these tags from being identified. The second issue is to forward previously read tag information towards a decision center responsible for triggering appropriate responses using the deployed infrastructure, in this case RFID readers. Both issues are tackled in this paper by proposing a crosslayer solution taking account of the density and mobility of both readers and tags to offer the best performance. Our proposal is inspired by previous works in anti-collision algorithms for RFID readers, adapts and combines these latter to enable the multihop wireless gathering of data. Data gathering relies on reader activity to avoid interfering with this action and their relative positions [10]. The new proposal is however enhanced with data-priority functionality, providing a differentiation between critical and standard data. Literature approaches only consider the hop count towards the sink as a metric, which in turn would affect the network load as well as radio activity for a subset of nodes used as forwarding hops and consequently the number of retrials to access the medium. Unlike these approaches, we rely on fuzzy logic to combine several metrics and provide a solution with good overall performance. 
Our solution features the following characteristics :

- distributed and local: all readers run the same algorithm only based on the local data;

- fair: medium access is fair and radio resource is not monopolized;

- reliable: providing a trustworthy data rate at the base station;

- low latency: ensuring the information read on the tag is forwarded towards the base station as fast as possible; - data priority aware: able to differentiate critical and data and route them with regards to requirements in terms of data rate and latency;

- compliant: our algorithm can be combined with any distributed TDMA anti-collision algorithms.

Our solution reaches above $96 \%$ of successful delivery rate for standard packets and close to $100 \%$ for priority ones, reducing latency up to $13 \%$ for priority packets compared to standard ones.

The rest of this paper is organized as follows: Section II provides a review of the state of the art regarding tackled issues, Section III presents the assumptions made regarding the hardware used and reveals the steps maintained from previous works. Section IV details the mechanism used by our proposal before it is evaluated in Section V. Finally Section VI concludes the work by providing future research directions.

\section{RELATED WORK}

In this section, we review the main literature work in the two issues raised in Section I : reading collisions and data routing. To the best of our knowledge, there is no work considering both issues jointly as we do.

\section{A. Reader anticollision}

The first issue addressed in RFID systems was reading collisions. They can happen at different levels and have been the main topic of research in the late years. Tag-totag collisions happen when multiple tags are interrogated by the same reader at the same time. To correct this issue several proposals were made and can be found classified in [11]. Reader-to-reader collisions happen when several readers attempt to read the same $\operatorname{tag}(\mathrm{s})$ at the same time. Their interrogation signals collide over the corresponding $\operatorname{tag}(\mathrm{s})$ which are then not identified properly. These are the type of collisions that will be addressed in the rest of the paper. Both TDMA and CSMA proposals were made to resolve this issue. Regarding distributed TDMA algorithms, several variations of Colorwave [12] were made with readers randomly choosing a timeslot within a defined range. Upon collision (readers in the same vicinity choosing the same timeslot), readers chose a new timeslot and send a kick message to advertise their new timeslot selection. Based on the number of kick messages recorded, readers can adapt the way they chose their following timeslots [13], [14]. Distributed CSMA algorithms like [15], uses beacon exchanges between readers to estimate the distance between them and provide a compromise over colliding tags. As such, readers decide whether to read or not. Tag information is then shared among readers. But such a solution is prone to errors due to distance estimation.

In [16], we proposed two distributed TDMA anticollision algorithms that consider both high deployment density and mobility of both readers and tags. mobile-Distributed Efficient \& Fair Anti-collision for RFID protocol (mDEFAR) make readers randomly chose timeslots and broadcast beacons before accessing the medium. Management of priority levels allows to increase fairness in medium access. Coverage Oriented Reader Anti-collision (CORA) also has readers randomly select timeslots and broadcast beacons but every reader records the number of potential colliding and non-colliding neighbors and based on these numbers either reads or gets disabled. Using mobile readers and dense deployment, this solution increases the fairness and throughput of the system allowing for a quick coverage of all deployed tags in the reading range.

\section{B. Tag information gathering}

To the best of our knowledge, multihop tag gathering has not been investigated jointly with RFID reading. In [15], authors discuss an architecture of readers communicating with a base station for IoT applications but this feature is not further studied in the paper. In WSN literature, nodes are equipped with a long range communication interface allowing them to reach the base station. Nevertheless, with our already made assumptions regarding the density and mobility of deployment of readers, we figured these features should be taken as an advantage to provide a multihop routing scheme. Several authors working on Mobile Adhoc NETworks (MANET) proposed high performance multihop routing schemes for WSN [17]. But these proposals unfortunately cannot be directly applied as is to our study case of RFID systems because of two different actions (tag reading and data forwarding). Also in our RFID systems, readers send data based on their interrogation activity defined by the running anti-collision algorithm, which is not the case in most of the MANET proposals. Combining the activity scheduling and routing schemes allows the system to be more energy efficient and prevents overlapping of interrogation and forwarding phases.

\section{AsSUMPTIONS AND BACKGROUND}

\section{A. Assumptions}

Since readers are expected to both interrogate tags and forward their data towards a base station, we assume our readers are equipped with two radio interfaces. The first interface, with a range of $d_{C R T}$, is used to interrogate tags. The second interface, with a range of $d_{C o m}$ uses a different channel, which we consider not to interfere with tag interrogation, and has two purposes. It is used 
by readers to communicate with their reader neighbors to agree on their interrogation activities depending on the anti-collision algorithm chosen. This assumption has been made several times in the state-of-the-art [12], [13], [15], [18]. This interface will also be used for tag information forwarding. In order to allow readers the discovery of colliding neighbors, the values were set as follows: $d_{C o m}=2 \times d_{C R T}$.

\section{B. Background}

As stated in Section I, we intend to produce crosslayer anti-collision and tag information routing algorithms for RFID. As such, our solution should be based on either existing or new scheduling algorithms for RFID readers. In [10], we introduced how tag information forwarding could be added to the interrogation activity of readers. We will here quickly detail how the network topology is formed and maintained.

1) Network Initialization: Our proposals are planned for distributed RFID systems in which readers are unaware of their positions. As such, we proposed to construct a topology based on a gradient construction towards the sink. Each reader has a rank corresponding to the number of hops (forwarding readers) needed to reach the sink, obtained thanks to a hop-by-hop relay, and identifying as their parent the node that allows the shorter path to the sink. Fig. 1 shows how the topology is constructed with 22 readers $\{A ; \ldots ; F\}$ and a single sink. A random backoff is drawn before forwarding the beacon to avoid collisions which would leave some nodes apart from the topology. On Fig. 1, the number next to the reader name represents its rank and the thick lines represent the parent $_{i}$ for each node.

2) Interrogation Scheduling: In most distributed TDMA anti-collision algorithms, readers choose their timeslots randomly within a given range. In our proposal, readers choose a slot based on their ranks. Readers with an even (resp. odd) rank $\left(\operatorname{rank}_{i} \% 2=0\right)$, randomly choose an even timeslot number (resp. odd timeslot number) within $\left[0 ; \max \_\right.$rank $[$. Interrogation activity is organized in rounds during which readers contend only once using their chosen timeslot. This limits interrogation round size to round_size = max_rank and decreases potential collisions. Indeed, readers can now be in the same vicinity but on different ranks ensuring they do not choose the same timeslot. Once timeslots are chosen, readers perform their interrogation activities according to the chosen anti-collision algorithm. In Fig. 1, readers $\{A ; B ; C\} \cup\{I ; \ldots ; O\}$ have odd ranks and will choose odd numbered timeslots within $[0 ; 4[$ (resp. even).

3) Topology maintenance: Since we are considering IoT applications involving mobile RFID readers, the topology of readers keeps evolving and the rank and parent need to be updated. When a reader interrogates tags and needs to forward its data toward the sink, it broadcasts a beacon to its neighbors with: (i) its rank in the current topology $\left(r a n k_{i}\right)$, (ii) the number packets it needs to forward (containing either its own reading or the ones it is in charge to forward) $\left(\right.$ load $\left._{i}\right)$, (iii) the radio activity in its vicinity (number of beacons and packets exchanged) $\left(\right.$ radio $\left._{i}\right)$ and (iv) the number of backoff trials it went through to forward its data during previous rounds (backof $f_{i}$ ). Upon reception of such a beacon, neighboring readers update their own rank rank $_{i}$ and parent $_{i}$ accordingly with the value of $\operatorname{rank}_{R X}$ contained in the beacon.

\section{PROPOSALS}

After reading tags and determining whether or not the information is critical or priority, readers have to forward the data towards a sink. If data is labeled as standard, the reader will send it to its parent $_{i}$ as computed in the initialization phase. Otherwise, if the data is determined as priority, it will be forwarded using parentprioi determined by the combination of several metrics using fuzzy logic.

\section{A. Fuzzy Logic}

Fuzzy logic [19] aims to develop a conceptual framework for systems that are too complex or ill-defined to produce precise quantitative analysis. It allows the translation and combination of several complex elements into more accessible fuzzy sets in order to produce a simpler, yet efficient output variable. A fuzzy logic system is constructed in three steps:

1) Fuzzification: Fuzzification converts data into fuzzy sets using membership functions (triangular, singleton, bell, etc.). In our case, readers will convert the values of each metric $\mathrm{m}=\left\{\operatorname{load}_{R X}, \operatorname{radio}_{R X}\right.$, backof $\left.f_{R X}\right\}$ received from neighbors into 3 fuzzy sets according to the following statements:

- if $m<$ low $_{m}$ the metric is translated as Excellent; - if $l o w_{m} \leq m<u p_{m}$ the metric is translated as Good; - if $m \geq u p_{m}$ the metric is translated as Bad.

$l_{o} w_{m}$ and $u p_{m}$ are two thresholds set for each considered metric $m$ (load, radio and backoff) based on made observations or expected behavior.

2) Definition of fuzzy rules: The fuzzy rules combine the different fuzzy sets through simple "IF ... THEN ... ELSE ..." statements. They determine the relationship between the fuzzy sets and the desired output variable. Based on the fuzzy sets obtained from fuzzification, we proposed the set of rules expressed in Table I. We aim to reduce network load, consequently the corresponding fuzzy set was chosen as a main ruler.

3) Defuzzification: Defuzzification is the last step of the process during which the obtained output variable is translated into a value used for decision making. In our case, the cardinal values from Table I $1 ; 2 ; 3 ; 4 ; 5$ will be used to determine the preferred parentprio $i$ used to forward priority packets. 


\begin{tabular}{c|c|c|c}
\multicolumn{3}{c|}{ Fuzzy sets } & Output \\
\hline Load & Radio & Backoff & Metric \\
\hline Excellent & Excellent & Excellent & 1 \\
Excellent & Good/Bad & Good/Bad & 2 \\
Good & Excellent & Excellent & 2 \\
Good & Good/Bad & Good/Bad & 3 \\
Bad & Excellent & Excellent & 4 \\
Bad & Good/Bad & Good/Bad & 5
\end{tabular}

TABLE I: Definition of fuzzy rules

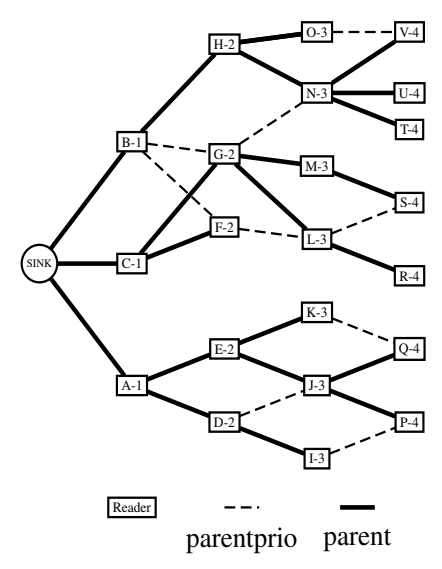

Fig. 1: Network example

\section{B. Data priority awareness}

Depending on the status of the data, the next hop to forward will either be its parent ${ }_{i}$ based on hop count to Sink or its parentprio ${ }_{i}$ determined by the previously stored metrics combined through fuzzy logic. Since readers are already separated by their rank and timeslots, readers that read during slot $n$ can transmit their data during slot $n+1$. The interrogation channel being different from the dedicated communication channel, both activities can take place without interference. While readers with even timeslots on even rank contend and interrogate tags, readers on odd timeslots and rank can forward their data and inversely.

1) Forwarding using parent $_{i}$ : In this case, the data to forward is standard and can follow the hop count. As such, based on all beacons received from neighbors, the $i^{\text {th }}$ reader forwards its data to its parent $t_{i}$ determined by the hop count to the sink. Depending on the density, readers may have one or several potential forwarding nodes. However, just one will be chosen as parent $_{i}$. On Fig. 1, readers will send their standard data through the node linked with the thick line corresponding to the number of hops towards the sink.

2) Forwarding using parentprio ${ }_{i}$ : In this case, the data has been determined as critical and needs to be forwarded using the stored metrics from neighbors. The combination of these metrics is performed using fuzzy logic and the three steps described in Section IV-A.
Based on all received beacons from neighbors, i-th reader is able to compute the output variable for each forwarding node in range (see Algorithm 1). The forwarding node with the smallest metric will be chosen as parentprio $_{i}$ and used to forward priority packets. On Fig. 1, the dashed lines represent the parentprio $_{i}$ links. For example, readers $\mathrm{F}$ and $\mathrm{G}$ despite having parent $_{F ; G}=C$ depending on the current network load, radio activity and backoff trials of $\mathrm{C}$ and $\mathrm{B}$ will chose parentprio $_{F ; G}=B$. However, some nodes share the same parentprio $_{i}=$ parent $_{i}$, this is the case for nodes $\{A ; B ; C\}$ that are only linked to the SINK, but also but also for other nodes like $\{H ; E ; D ; \ldots\}$.

Upon reception of a priority packet, the forwarding node

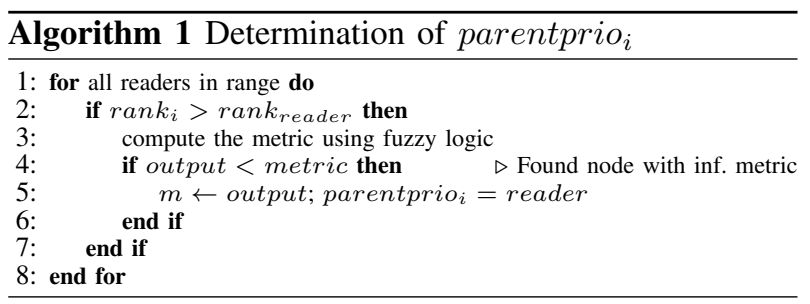

performs the same algorithm to select its parentprio $i$ and forward the packet toward the Sink.

\section{Evaluation}

In order to evaluate our proposals, we run simulations using WSNet which is an event-driven simulator for wireless sensor networks. According to the applications referred to in Section I, we considered two scenarios. The first one is a warehouse of $100 \times 100 \mathrm{~m}^{2}$ with 4000 tags disposed on 20 shelves and 80 mobile readers moving between aisles at a speed of $[0.5-1.5] \mathrm{m} / \mathrm{s}$. A single sink is considered for this scenario which is set at the center of the map. The second one is a part of a city of size $555 \times 555 \mathrm{~m}^{2}$ with 3000 static tags attached to urban infrastructures and mobile ones roaming the streets at a speed ranging between $[21-36] \mathrm{km} / \mathrm{h}$. 570 statics readers disposed at street corners and mobile ones roaming the street at a speed ranging between $[14-20] \mathrm{km} / \mathrm{h}$. The size of this map allowed us to verify the behavior of our proposals with several sinks in range, as such, 5 sinks were deployed.

We chose to deploy our cross-layer proposal on two previous anti-collision algorithms from the literature CORA and mDEFAR [16]. Their anti-collision results are not presented here since the modification added to these algorithms did not affect their performance regarding that aspect. They perform similarly to results obtained in [16], [20].

The values of $l o w_{m}$ and $u p_{m}$ were respectively set to $\{3 ; 6\}$ for load, $\{10 ; 20\}$ for radio and $\{20 ; 60\}$ for backoff. The values were chosen after several simulations which shown the best results using these sets. Figures 2a and $2 \mathrm{~b}$ respectively show the packet data ratio results 

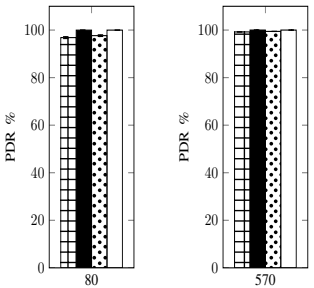

(a) PDR - (b) PDR - Ur-

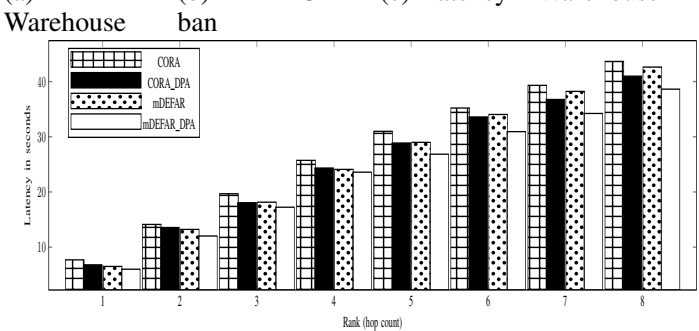

(d) Latency - Urban

Fig. 2: Data gathering results

in the warehouse and urban scenarios. The packet data ratio is the ratio of packets that successfully reached the $\operatorname{sink}(\mathrm{s})$ over the total number of packets sent. In the warehouse (see Figure 2a) we can see that results are over $96 \%$ with algorithms. Regarding standard packets, CORA records slightly lower performance (96.83\%) due to the higher number of messages that are created compared to mDEFAR (97.66\%). Indeed CORA offers a higher throughput and reads more tags than mDEFAR, hence more data needs to be forwarded creating packet loss. Priority packets are however delivered with a Packet Delivery Rate (PDR) close to $100 \%$ with both CORA $(99.97 \%)$ and mDEFAR (99.98\%). In the urban scenario, results are better since both more sinks and readers are available for nodes to forward their data to. With both CORA and mDEFAR $100 \%$ of priority packets are successfully received at sinks. Figures $2 \mathrm{c}$ and $2 \mathrm{~d}$ respectively show the latency results relatively to the rank of emitting reader in the warehouse and urban scenarios. The latency is expressed as the endto-end delay needed to forward data from the reader that interrogated the tag all the way to the sink. In the warehouse (see Figure 2c), we can see that using our fuzzy logic metrics we are to gain approximately $9-13 \%$ on each rank. The gain is lower at lower ranks since there are less potential forwarding nodes and parent $_{i}=$ parentprio $_{i}$ for some readers. Same observation is made in the urban scenario (see Figure 2d) where the gain is around $8-12 \%$.

\section{CONCLUSION}

This paper presents a cross-layer anti-collision and routing algorithms for RFID systems in IoT applications. Performance evaluation of our proposal shows the benefit of our fuzzy logic approach to combine several metrics in order to improve network load and reduce latency needed for the delivery of priority packet compared to standard ones. Next steps will look into the effects of defining different fuzzy rules and how they can affect latency and network load. Experimental evaluation is also considered to further confirm these results.

\section{REFERENCES}

[1] K. Finkenzeller, RFID Handbook: Fundamentals and Applications in Contactless Smart Cards and Identification. John Wiley \& Sons, 2003, ch. 3, pp. 29-61.

[2] ETSI, "Radio frequency identification equipment operating in the band $865 \mathrm{mhz}$ to $868 \mathrm{mhz}$ with power levels up to $2 \mathrm{w}$ and in the band $915 \mathrm{mhz}$ to $921 \mathrm{mhz}$ with power levels up to 4 $\mathrm{w}$; harmonised standard covering the essential requirements of article 3.2 of the directive 2014/53/eu," 2016.

[3] S. Kim, R. Vyas, J. Bito, K. Niotaki, A. Collado, A. Georgiadis, and M. M. Tentzeris, "Ambient rf energy-harvesting technologies for self-sustainable standalone wireless sensor platforms," Proceedings of the IEEE, vol. 102, 2014.

[4] D. J. Yeager, P. S. Powledge, R. Prasad, D. Wetherall, and J. R. Smith, "Wirelessly-charged uhf tags for sensor data collection," in Proc of IEEE Int. Conf. on RFID. IEEE, 2008.

[5] L. Catarinucci, R. Colella, and L. Tarricone, "Enhanced uhf rfid sensor-tag," IEEE Microwave and Wireless Components Letters, vol. 23, 2013.

[6] I. Zalbide, E. D’Entremont, A. Jiménez, H. Solar, A. Beriain, and R. Berenguer, "Battery-free wireless sensors for industrial applications based on uhf rfid technology," in Proc. of IEEE SENSORS. IEEE, 2014.

[7] M. Bartholmai, M. Stoppel, S. Petrov, and S. Hohendorf, "Two application examples of rfid sensor systems-identification and diagnosis of concrete components and monitoring of dangerous goods transports," ACTA IMEKO, vol. 4, 2015.

[8] B. Chowdhury and M. U. Chowdhury, "Rfid-based realtime smart waste management system," in Proc. of Australasian Telecommunication Networks and Applications Conference (ATNAC). IEEE, 2007.

[9] X. Jia, Q. Feng, T. Fan, and Q. Lei, "Rfid technology and its applications in internet of things (iot)," in IEEE CECNet, 2012.

[10] A. A. Mbacké, N. Mitton, and H. Rivano, "Data gathering solutions for dense rfid deployments," in IEEE UIC, 2017.

[11] K. K. Dheeraj, C. Kwan-Wu, and R. Raad, "A survey and tutorial of rfid anti-collision protocols," IEEE Communications Survey \& Tutorial, vol. 12, no. 3, 2010.

[12] J. Waldrop and D. W. Engels, "Colorwave: an anticollision algorithm for the reader collision problem," in ICC, 2003.

[13] F. Gandino, R. Ferrero, B. Montrucchio, and M. Rebaudengo, "Dcns: An adaptable high throughput rfid reader-to-reader anticollision protocol," IEEE TPDS, vol. 24, no. 5, 2013.

[14] M. V. Bueno-Delgado and P. Pavón-Mariño, "A maximum likelihood-based distributed protocol for passive rfid dense reader environments," The Journal of Supercomputing, vol. 64, 2013.

[15] H. Rezaie and M. Golsorkhtabaramiri, "A fair reader collision avoidance protocol for rfid dense reader environments," Wireless Networks, 2017.

[16] A. A. Mbacke, N. Mitton, and H. Rivano, "Rfid anticollision in dense mobile environments," in Proc. of IEEE WCNC, 2017.

[17] J. Loo, J. L. Mauri, and J. H. Ortiz, Mobile ad hoc networks: current status and future trends. CRC Press, 2016.

[18] V. Bueno-Delgado, R. Ferrero, F. Gandino, and P. Pavon-Marino, "A geometric distribution reader anti-collision protocol for RFID dense reader environments," IEEE T-ASE, 2013.

[19] L. A. Zadeh, "Outline of a new approach to the analysis of complex systems and decision processes," IEEE TSMC, 1973.

[20] A. A. Mbacke, N. Mitton, and H. Rivano, "Rfid reader anticollision protocols for dense and mobile deployments," MDPI Electronics SI "RFID Systems and Applications", 2016. 